\title{
A Study of Rapid Leishman Stain on Peripheral Blood Smear
}

\author{
Prem Kumar Essgir and Hemalatha Anantharamaiah* \\ Department of Pathology, Sri Devaraj Urs Medical College, Sri Devaraj Urs Academy of Higher Education and \\ Research, Tamaka, Kolar, Karnataka, India
}

\begin{abstract}
Background: Romanowsky stains are universally used for routine staining of peripheral blood smears. Among these Leishman stain is most commonly used in hematology laboratories worldwide. This study was done to stain peripheral blood smears with modified Leishman stain (MLS) on Day 1, Day 5, Day 10 of preparation of the stain and to assess the quality of staining by scoring the stained smears by calculating the Quality Index (QI) and comparing the scores with normal peripheral smear.
\end{abstract}

Methods: Study was done in the hematology section of our institute from December 2016 to February 2017 on a sample size of hundred and one. (MLS) was prepared by adding phenol to the Leishman stain. All cases were stained with modified Leishman stain on day 1, 5 and 10 after preparing the stain. All the smears were scored based on overall staining, cytoplasmic staining, nuclear morphology, red cell staining and platelet staining. Quality index was calculated by dividing the score obtained by maximum score possible.

Result: Overall staining, cytoplasmic staining, nuclear morphology, red cell staining and platelet staining were better on day 10 after preparation of MLS when compared to day 1 and day 5. The Quality Index of stained smears normal leishman stained smear was 0.95 , score on day 1 of preparing stain was 0.71 , score on day 5 was 0.73 and day 10 of preparing stain was 0.89 .

Conclusion: MLS can be used for staining of thin peripheral blood smears in 4 minutes, unlike the conventional Leishman stain method which takes about 10-15 minutes.

Keywords: Modified Leishman Stain, Quality Index, Peripheral Blood Smear

\section{Introduction}

A Romanowsky stain contains both acidic and basic dyes, and is universally used for staining peripheral blood smears in various laboratories. Original Romanowsky method was modified by William Boog Leishman a British pathologist and was called as Leishman's stain. ${ }^{1}$ The advantage of Leishman stain over Giemsa staining (another Romanowsky stain used in hematology) is that it can be prepared easily, low cost and staining can be done within 10-15 minutes. ${ }^{2,3,4}$ Though many modifications of different Romanowsky stains have been done, only few modifications of Leishman stain has been introduced to reduce the duration of staining of peripheral blood smear. ${ }^{5}$

Phenol, an accentuating agent (also used in cytogenetics) is known to reduce the duration of staining when added to Leishman stain. ${ }^{6}$ Hence this study was taken up to assess the effectiveness of modified Leishman stain (MLS) in staining the smear on day 1,5 and 10 of preparing the stain.

\section{Materials and Methods}

The present study was done in the hematology section of our Laboratory from December 2016 to February 2017. Before starting the study Ethical clearance was obtained from the Institutional Ethical Committee. Three hundred and three smears made from 101 samples received in our laboratory were included in our study. Normal smear was prepared and stained as a part of regular diagnostic procedure and they were numbered and stored after routine reporting.

Cases with scanty left out blood sample, blood samples from immune compromised individuals and clotted samples were excluded from study. Informed consent was taken from all the cases. Two $\mathrm{ml}$ of blood was collected into K3 EDTA (Ethylene Diamine TetraAcetic acid) tube for routine diagnostic procedure and identification number was given by bar coding system in phlebotomy collection area and the blood tubes were transported to hematology section for processing according to our laboratory policy.

Hundred and one cases were divided into 7 batches. Each batch had 15 consecutive cases received on a particular day. Three smears were made from each of these samples. The day of smear preparation of that batch was considered as day 1. Fresh modified Leishman stain was made on that day. And same stain was used for staining one batch of slides on day 1, 5 and 10 . The same procedure was repeated for all batches. Last batch had only eleven cases. This batch analysis was followed for convenience of the investigator. 
After performing the necessary investigations 3 smears were made from left over sample of each case by wedge technique and labelled. All smears were labeled as $1 \mathrm{a}, 1 \mathrm{~b}$ and $1 \mathrm{c}$ for case $1,2 \mathrm{a} 2 \mathrm{~b}$ and $2 \mathrm{c}$ for case 2 for case 2 and so on. "a" indicated day 1 , "b" indicated day 5 and "c" indicated day 10 of staining. Smears stained by normal Leishman stain was labeled by unique identification of our laboratory.

Preparation of Modified Leishman Stain:: Hundred and Fifty milligrams of Leishman powder was weighed using electronic balance and dissolved in $100 \mathrm{ml}$ of methanol in a glass beaker using a magnetic stirrer for five minutes. To this mixture 50 microliter of liquefied phenol was added and mixed for another five minutes. The contents were transferred into dark bottle and used on day 1,5 and 10 of each batch. The concentration of phenol used and duration of staining, the days on which the staining of smears were done was based on a pilot study that was done on 10 cases.

Staining of smears: Modified Leishman stain and buffered water was taken in coplin jar Air dried blood smear were labeled was placed in rapid Leishman stain for 1 minute After 1 minute slides were removed from stain and placed in coplin jar containing buffer water ( $\mathrm{pH}$ 6.8) for 3 minutes.

After 3 minutes slides were then dried on slide rack After staining all 303 smears (101 cases $\mathrm{x} 3$ smears), all the slides were mixed and these 404 slides [numbered from $1-101$ $(a, b, c)$ and the smear stained with normal Leishman stain that was used for reporting] were then renumbered from 1 to 404 . A master copy of the smear numbers (1 to 404) and corresponding case number and day $(1,1 \mathrm{a}, 1 \mathrm{~b} 1 \mathrm{c})$ was maintained. These smears were observed by both authors independently and score was given.

Scoring of smears was given based on five parameters of overall staining, cytoplasmic staining, nuclear staining, $\mathrm{RBC}$ staining, platelet staining.

Score $0=$ Unsatisfactory $/$ Unstained, Score $1=$ Satisfactory, Score 2 = Excellent

The maximum score for a single case was calculated taking into account all parameters and it was 10 . The overall maximum possible score in the study was calculated by multiplying the number of cases by 10. QI (Quality index) of the stain was obtained by ratio of actual score obtained/ maximum score possible.

Since our study was based on interpretation of staining characteristics no statistical instruments were used. Instead the efficacy of staining was assessed based on scoring the staining characters of the smears

\section{Result}

A total of 101 cases were included in our study. Maximum number of cases were in the age group 20-29 years age (26.7\%). Next common age group was 30-39 years age 69 and 60-69 years age (15.8\%). Least number of cases belonged to age group $0-9$ years age (4.9\%). Out of the 101 cases 44 were male and 57 were female.

The Quality Index (QI) of day 10 was 0.89 , whereas day 1 was 0.71 and day 5 was 0.73 . The overall staining, cytoplasmic staining, nuclear morphology, red cell staining and platelet staining was better on day 10 as compared to day 1 and day 5. It was close to scoring of normal smears (0.95). Figure 1,2,3 shows the appearance of smears stained by modified Leishman stain on day 1,5 and 10 respectively.

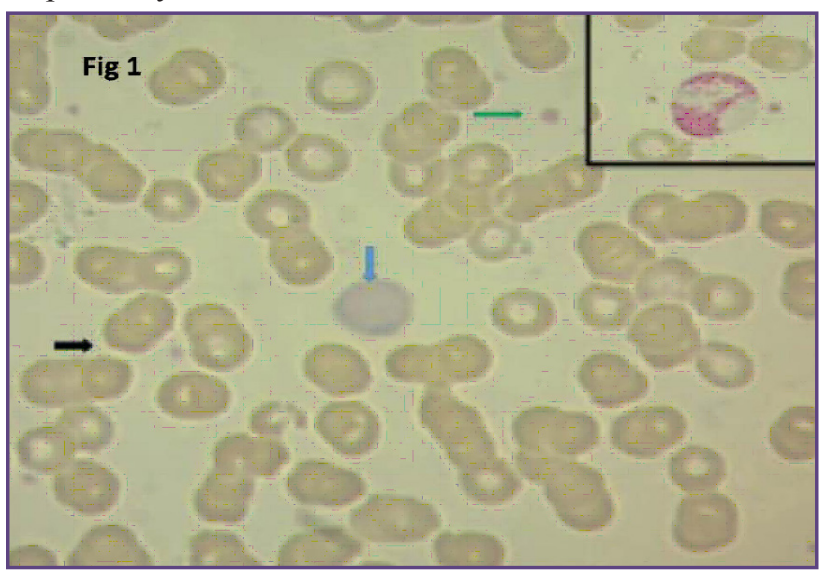

Fig. 1: Peripheral blood smear showing blue-green red cells (Black arrow), poorly stained leucocytes (blue arrow) and poorly stained platelets in the background (Green arrow). Inset shows poorly stained eosinophil. Modified Leishman stain on Day 1 (x1000 magnification).

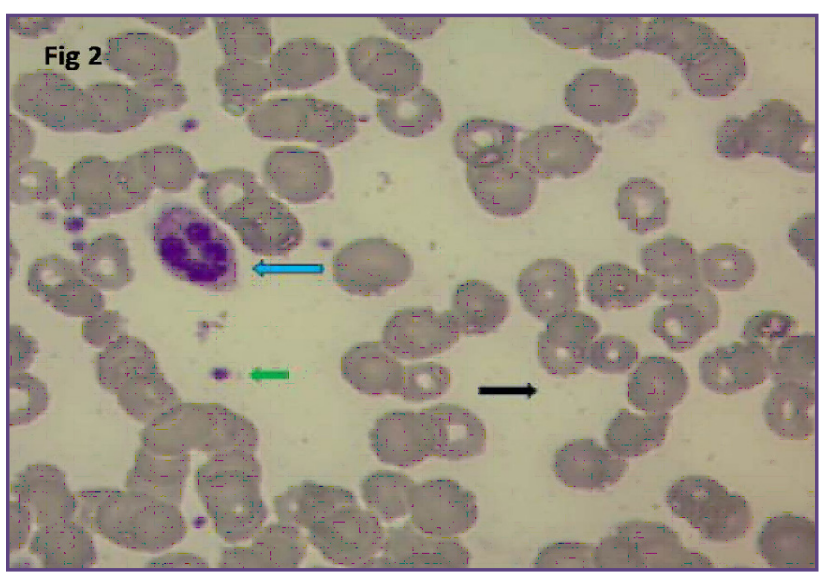

Fig. 2: Peripheral blood smear showing Blue-green red cells (Black arrow), well stained leucocytes (blue arrow) and platelets in the background (Green arrow). Modified Leishman stain on Day 5. (x1000 magnification). 


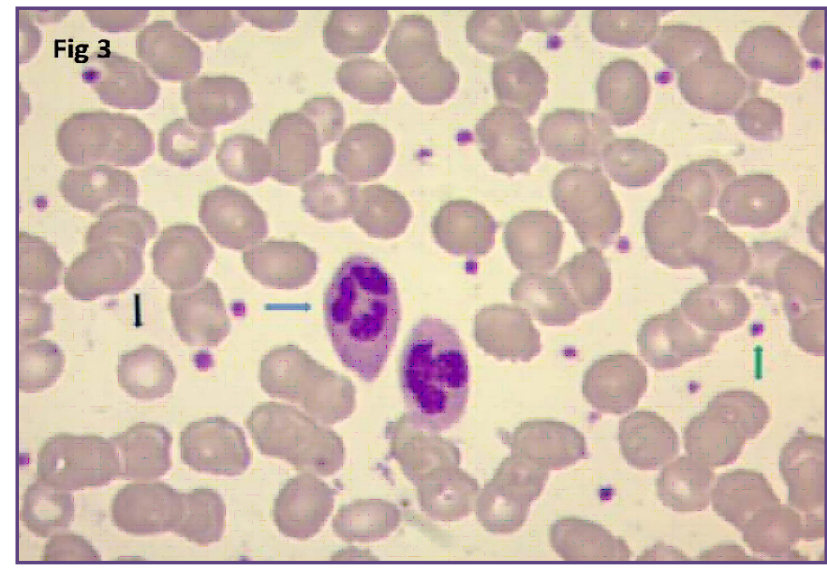

Fig. 3: Peripheral blood smear showing distinctly stained red cells (Black arrow), leucocytes (blue arrow) and platelets in the background (Green arrow). Modified Leishman stain on Day 10. (x1000 magnification).

\section{Discussion}

Leishman stain is composed of acidic dye and a basic dye. Basic dye (Methylene blue or Azure B) is positively charged and binds to anionic sites and imparts blue gray color to nucleic acids, nucleoproteins and granules of basophils. Acidic dye (Eosin Y) is negatively charged and binds to cationic sites and imparts orange red color to hemoglobin and eosinophil granules. ${ }^{7,8}$ Both the dyes are dissolved in acetone free methyl alcohol (methanol). It is a water insoluble stain and takes around 10 minutes for staining of thin blood films.

In the era of rapid diagnosis of diseases and use of automated hematology analyzers a need arises for reducing the duration staining process of peripheral blood smear, more so in cases of sepsis, acute leukaemias, chronic leukemias and thrombocytopenia.

Use of rapid stains in diagnostic laboratory started in 1941 by John William Field who introduced rapid stain for thick blood films for identification of hemoparasites. ${ }^{9}$ Recently rapid 5 minute Giemsa stain has also been used to visualize and diagnose various disease conditions. ${ }^{10}$

Fasakin et al modified the Leishman stain by adding phenol to Leishman stain for rapid analysis of staining of peripheral blood smears. He concluded that modified Leishman stain can be used to reduce staining time with overall reduction in turnaround time (TAT) on peripheral blood smear reporting. He also prepared ratio of phenol:leishman powder as 1:5 1:4 1:3 1:2 and 1:1 respectively in $100 \mathrm{ml}$ of staining solution, and concluded that $1: 5$ and $1: 3$ ratio of modified Leishman stain gives better morphology than the conventional Leishman stain. ${ }^{11}$ Phenol is used as an accentuating agent in Ziehl Neelsen stain. The role phenol is critical in this stain. ${ }^{12}$
When phenol is added to leishman stain it influences the fixation, uptake of stain, and facilitating rapid cellular analysis of blood without alteration in morphology of blood cells.

Both phenol and methanol are organic compounds with terminal hydroxyl functional group.

Phenol when dissolved in methanol facilitates their reactivity to be shortened, hence the overall time taken for fixation and staining is reduced. Based on the study by Fasakin et al, we did a pilot study on 10 cases of peripheral blood smear using phenol and Leishman powder + methanol in a ratio of 1:5. We decided to include day 1 , day 5 and day 10 based on staining quality of smears. The changes on day $2,3,4,6,7,8,9$ was subtle and was comparable to either day 1 , day 5 and day $10 .{ }^{11}$

Since no other studies has been done in a similar way, we used quality indicator to compare and assess the staining quality of overall staining, leucocyte staining (nuclear and cytoplasmic), erythrocytes staining and platelet staining on day 1 , day 5 and day 10 and compare it with normal stained smear.

Though smears stained on all three days were satisfactory. Some of the smears stained on day 1 slides appeared bluish black color macroscopically and microscopically red cells appeared bluish-green color,leucocytes (granules and nuclei) appeared poorly stained and platelets were poorly stained (see fig.1).

Some of the smears stained on day 5 showed bluish green red blood cells and poorly stained granules of the leucocytes, but were less as compared to day 3 . In some of the smears stained on day 10 only few red cells appeared light bluish color but leucocytes and platelets stained well. Hence the modified Leishman stain used on day 10 of preparation gave better results as compared to day 1 and day 5 .

Drawback of Modified Leishman stain: Some parts of smear which were thick appeared bluish-black color when stained with modified Leishman stain. Red cells at those places appeared bluish-green color and White blood cells and platelets were poorly stained in those areas.

\section{Conclusion}

In this study we compared efficacy of modified Leishman stain on day 1, day 5 and day 10 of stain preparation. Smears stained on day 10 had better staining characteristic with Quality Index (QI) of 0.89 as compared to day 1 is 0.71 and day 5 is 0.73 and was as good as normal Leishman stained peripheral smear. 
Modified Leishman stain can be used in staining of thin peripheral blood smears within 4 minutes.

\section{Reference}

1. Bain, Barbara \& Mitchell Lewis, S. Preparation and staining methods for blood and bone marrow films. Dacie and Lewis Practical Haematology. 11th ed.Amsterdam: Elsevier health sciences; 2011. p.59.

2. Sathpathi S, Mohanty A, Satpathi P, Mishra S, Behera P, Patel G et al. Comparing Leishman and Giemsa staining for the assessment of peripheral blood smear preparations in a malaria endemic region in India. Malaria J 2014;13(1):512.

3. Wittekind D. On the nature of Romanowsky dyes and the Romanowsky-Giemsa effect. Clinical \& Laboratory Haematol 2008;1(4):247-262.

4. Woronzoff-Dashkoff KP. The Erlich-Cheminsky-GrumwaldLeishman-Ructer-Wright- Giemsa-Lillie-Roe-Wilcox stain. The mystery unfolds. Clin Lab Med. 1993;13:759-771

5. Bain, Barbara \& Mitchell Lewis, S.. Preparation and staining methods for blood and bone marrow films. In; Dacie and
Lewis Practical Haematology. 11th ed.Amsterdam: Elsevier health sciences; 2011. p.62.

6. Weber M, Weber M, KleineBM.P.In: Ullmann'sencyclopedia of industrial chemistry. 7th ed, Weinheim:Wiley-VCH;2004.

7. Wittekind D, Kretschmer V, Sohmer I. Azure B-eosin Y stain as the standard Romanowsky-Giemsa stain. British J Hematol 1982;51(3):391-393.

8. Mathur A, Tripathi A, Kuse M. Scalable system for classification of white blood cells from Leishman stained blood stain images. J Pathol Informatics 2013;4(2):15.

9. K.D. Chatterjee, Examination of blood for parasites, In: Parasitology.13 th ed, CBS;2015.

10. Jager MM, Murk JL, Piqué RD, Hekker TA, VandenbrouckeGrauls CM. Five-minute Giemsa stain for rapid detection of malaria parasites in blood smears. Trop Doct. 2011 ;41(1):33-5.

11. Fasakin KA, Okogun, Omisakin CT, Adeyemi AA, Esan British AJ. Modified Leishman Stain: The Mystery Unfolds. J Med Medical Res 2014; 4(27): 4591- 4606.

12. Lamana, C. The nature of the acid-fast stain. J Bacteriol $1946 ; 52: 99-103$.

*Corresponding author:

Dr. Hemalatha A, Associate Professor, Department of Pathology, Sri Devaraj Urs Medical College, Tamaka, Kolar 563101 India Phone: +91 9972212324

Email: drhemashashi@gmail.com

Financial or other Competing Interests: None. 